\title{
Core-Guided Minimal Correction Set and Core Enumeration
}

\author{
Nina Narodytska ${ }^{1}$, Nikolaj Bjørner ${ }^{2}$, Maria-Cristina Marinescu ${ }^{3}$, Mooly Sagiv $^{1,4}$ \\ ${ }^{1}$ VMware Research, USA \\ ${ }^{2}$ Microsoft Research, USA \\ ${ }^{3}$ Barcelona Supercomputing Center, Spain \\ ${ }^{4}$ Tel Aviv University, Israel \\ nnarodytska@vmware.com,nbjorner@microsoft.com, maria.marinescu@bsc.es, msagiv@acm.org
}

\begin{abstract}
A set of constraints is unsatisfiable if there is no solution that satisfies these constraints. To analyse unsatisfiable problems, the user needs to understand where inconsistencies come from and how they can be repaired. Minimal unsatisfiable cores and correction sets are important subsets of constraints that enable such analysis. In this work, we propose a new algorithm for extracting minimal unsatisfiable cores and correction sets simultaneously. Building on top of the relaxation and strengthening framework, we introduce novel techniques for extracting these sets. Our new solver significantly outperforms several state of the art algorithms on common benchmarks when it comes to extracting correction sets and compares favorably on core extraction.
\end{abstract}

\section{Introduction}

A set of constraints is over-constrained if there is no solution that satisfies these constraints. Over-constrained problems often occur in practical applications, including verification, configuration, diagnosis, etc., [Belov et al., 2012; Liffiton and Sakallah, 2008b; Junker, 2004]. For an over-constrained problem, it is important to understand why the problem's constraints are inconsistent and how we can relax constraints to eliminate these inconsistencies. To do so, two informative notions were introduced in the literature [Reiter, 1987; Liffiton and Sakallah, 2008a; Liffiton et al., 2016]. The first notion is an unsatisfiable core. An unsatisfiable core is a subset of constraints that is unsatisfiable regardless of the rest of the formula. We are interested in minimal unsatisfiable cores (MUSes) under the set inclusion relation as they represent a concise description of the problem's inconsistencies. Another important notion is a correction set, which is a subset of clauses removal of which makes the remaining formula satisfiable. Again, minimal correction sets (MCSes) are interesting for a user as we want to satisfy as many constraints as possible in the remaining formula. As minimal cores and correction sets are not unique, it is important to build tools and algorithms to enumerate them. Applications in verification and knowledge representation rely on the ability to enumerate these sets [Liffiton and Sakallah, 2008b].

Several new algorithms have been developed during the past decade for efficient MCS or/and MUS enumeration. They can be roughly divided into two classes. The first class of algorithms works with the original set of constraints and explores a lattice of all possible subsets of constraints efficiently to search for cores or/and correction sets [Liffiton and Sakallah, 2008a; Marques-Silva et al., 2013; Bacchus and Katsirelos, 2015; Bacchus et al., 2014; Zielke and Kaufmann, 2015; Bacchus and Katsirelos, 2016; Zhao and Liffiton, 2016; Previti et al., 2018]. The second class modifies the formula at each step and extracts minimal correction sets from the modified formula [Morgado et al., 2012; Marques-Silva and Planes, 2008; Liffiton and Sakallah, 2009; Alviano, 2017b]. The algorithms in this class are based on MAXSAT solvers so we call this class the relaxation and strengthening framework as it iteratively transforms an unsatisfiable formula to satisfiable and vice versa. Our algorithm exploits ideas from both classes. We work within the relaxation and strengthening framework but we also keep information about the part of the lattice that we have explored so far. We extend this framework to perform core enumeration and propose a minimal correction set rotation technique that is based on ideas of model rotation used for minimization of cores [Belov and Marques-Silva, 2011].

In this work we advance state-of-the-art in MUS and MCS enumeration algorithms with the following main contributions. First, we develop an efficient MCS and MUS enumerator using the relaxation and strengthening framework. Second, we propose a new technique for MCS enumeration using a correction set rotation method. Our method allows checking minimality of a correction set efficiently without calling a SAT solver. Third, we propose a new blocking technique that maintains dependencies between relaxation variables and selector variables that enables MUS enumeration using the relaxation and strengthening framework. Finally, we preform evaluation in a prototype solver FLINT. FLINT is built on top of the PM1 core-guided MaxSAT solver [Fu and Malik, 2006]. We compare it with MARCO, the state-of-the-art method that simultaneously extracts MUSes and MCSes. We show that our approach outperforms MARCO in enumerating these objects. Moreover, our algorithm, geared toward enumerating MCSes, 
significantly outperforms a variant of MARCO tuned the same way and one of the best methods for MCSes enumeration on a standard set of benchmarks [Marques-Silva et al., 2013; Alviano, 2017b].

\section{Background}

Basic definitions. A satisfiability problem $\varphi$ consists of a set of clauses $\left\{C_{1}, \ldots, C_{m}\right\}$ over a set of Boolean variables $\operatorname{vars}(\varphi)$. A literal $l$ is either a variable $x \in \operatorname{vars}(\varphi)$ or its negation $\bar{x}$. A clause $C$ is a disjunction of literals $\left(l_{1} \vee \cdots \vee\right.$ $\left.l_{n}\right)$. An assignment $I$ of the variables $\operatorname{vars}(\varphi)$ is a mapping $\operatorname{vars}(\varphi) \mapsto\{0,1\}$. A clause $C$ is satisfied by an assignment, $I(C)=1$, iff $I(l)=1$ for some $l \in C$, otherwise $C$ is falsified by $I$ and $I(C)=0$. A set of clauses $\varphi$ is satisfied by an assignment, $I(\varphi)=1$, iff $I(C)=1$ for all $C \in \varphi$.

Cores and correction sets. We define two important objects of interest for unsatisfiable formulas: a minimal unsatisfiable core and a minimal correction set.

Definition 1 (MCS). A minimal correction set is a subset of clauses MCS $\subseteq \varphi$ such that $\varphi \backslash$ MCS is satisfiable and $\forall C \in \operatorname{MCS}:(\varphi \backslash \mathrm{MCS}) \cup\{C\}$ is unsatisfiable.

Definition 2 (MUS). A minimal unsatisfiable set is a subset of clauses MUS $\subseteq \varphi$ such that MUS is unsatisfiable and $\forall C \in$ MUS: MUS $\backslash\{C\}$ is satisfiable.

Example 1 (Running example). Consider a formula with five clauses: $\varphi=\left\{C_{1}, \ldots, C_{5}\right\}$, where $C_{1}=\left(x_{1}\right)$, $C_{2}=\left(\neg x_{1} \vee x_{2}\right), C_{3}=\left(\neg x_{2}\right), C_{4}=\left(\neg x_{1} \vee x_{3}\right)$, $C_{5}=\left(\neg x_{3}\right)$. We have two minimal unsatisfiable cores $\left\{\left(C_{1}, C_{2}, C_{3}\right),\left(C_{1}, C_{4}, C_{5}\right)\right\}$, and five minimal correction sets $\left\{\left(C_{1}\right),\left(C_{2}, C_{4}\right),\left(C_{2}, C_{5}\right),\left(C_{3}, C_{4}\right),\left(C_{3}, C_{5}\right)\right\}$.

During the enumeration procedure, we introduce relaxation variables, $b$, that are added to original clauses. For example, an original clause $C_{1}$ can be replaced with $C=\left(C_{1} \vee b\right)$, where $b$ is a relaxation variable. We denote $C$ without relaxation variables orig $(C)$ and the set of relaxation variables in the clause $C$ rel $(C)$. In this example, orig $\left(C_{1} \vee b\right)=\left\{C_{1}\right\}$ and $\operatorname{rel}\left(C_{1} \vee b\right)=\{b\}$.

Each MCS is a hitting set of MUSes if we look at each MCS as a set of clauses. Dually, each MUS is a hitting set of all MCSes [Reiter, 1987].

Power sets of clauses. [Liffiton et al., 2016] considered MUS/MCS enumeration as an exploration of the power set of all clauses in a formula. These subsets form a lattice by the subset relation. They introduce a notion of a map, which is a Boolean formula that encodes the lattice covered so far. To encode this formula, one selector variable $s_{i}$ for each clause $C_{i}$ is introduced, $S=\left\{s_{1}, \ldots, s_{n}\right\}$. Each time we encounter a subset of clauses $C^{\prime} \subseteq \varphi$ and learn that it is a core or a correction set, we update map using the following correction set blocking clauses (1) or core blocking clauses (2) ${ }^{1}$.

$C^{\prime}$ is a correction set $\operatorname{map}=\operatorname{map} \cup\left\{\vee_{C_{i} \in C^{\prime}} s_{i}\right\}$

$C^{\prime}$ is a core

$$
\operatorname{map}=\operatorname{map} \cup\left\{\vee_{C_{i} \in C^{\prime}} \bar{s}_{i}\right\}
$$

${ }^{1}$ In [Liffiton et al., 2016], the first constraint is defined for a complement of a correction set.
Example 2. Consider the instance from Example 1. We introduce five selector variables $\left(s_{1}, \ldots, s_{5}\right)$ as we have five clauses. Suppose we discover that a subset of clauses $\left\{C_{1}, C_{2}, C_{3}\right\}$ is a MUS. We update map by adding a new clause $\left(\bar{s}_{1} \vee \bar{s}_{2} \vee \bar{s}_{3}\right)$. Suppose we learn that $\left\{C_{1}\right\}$ is a MCS. We add a clause $\left(s_{1}\right)$ to map.

Note that map contains two types of blocking clauses. The first type contains clauses to block MCSes and the second type contains clauses to block MUSes. We call the former mapmes and the latter mapmus, map = mapmcs $\cup$ mapmus.

\section{Enumeration Algorithm}

\subsection{Overview}

Our algorithm is built on top of a framework proposed in [Marques-Silva and Planes, 2008; Morgado et al., 2012; Alviano, 2017b] that performs a sequence of relaxations and strengthenings of the formula to obtain MCSes. At a high level, it performs a sequence of rounds until a termination condition is met. Each round consists of two phases: Relax and Strengthen. Figure 1 provides a schematic representation of this framework. In the Relax phase, the algorithm starts with an unsatisfiable formula and weakens it by relaxing its cores until it becomes satisfiable. The first phase is, effectively, a call to a MaxSAT solver. The resulting satisfiable formula is passed to the second phase, Strengthen. In the Strengthen phase, solutions of the satisfiable formula are enumerated and are blocked making the formula unsatisfiable again. Relaxations and strengthenings ensures that these solutions are MCSes of the formula. Then the algorithm verifies the termination condition which checks whether all MCSes have been enumerated. If so, it comes to the final stage where the remaining MUSes are enumerated using hitting set duality between MCSes and MUSes. Otherwise, the algorithm iterates with another round of strengthening and relaxation. The existing algorithms differ in the underlying MAXS AT algorithms used to relax the formula and the way they block solutions to strengthen the formula.

Here we extend this framework in several ways. First, we show that with a new solution blocking technique we can extend this framework to MUS enumeration (RelaxCore and EnuMus procedures are highlighted using italic gray in Figure 1). Second, we show that we can exploit properties of the solutions to build an efficient MCS extractor (the MCSRotation procedure is highlighted using italic gray in 1). Finally, we prove correctness of the algorithm.

Algorithm 1 contains the main loop of the proposed enumeration algorithm EnuMMerator. We will describe our Relax and Strengthen functions below. We start by describing global variables and structures that we use. Relax and Strengthen modify a working formula $\psi^{d}$ and update global variables projs, map and cards that maintain necessary information about minimal cores and correction sets for the algorithm to operate. The set projs connects relaxation variables, $b$, and selector variables, $s$. Each time a new $b_{j}^{i}$ variable is added to a clause $C_{j}$, we connect $b_{j}^{i}$ with $s_{j}$ as $b_{j}^{i} \Rightarrow \bar{s}_{j}$. These constraints ensure that if $C_{j}$ is relaxed, i.e. any of its relation variables is 1 , then the corresponding selector variable $s_{j}=0$. The set map stores information about 


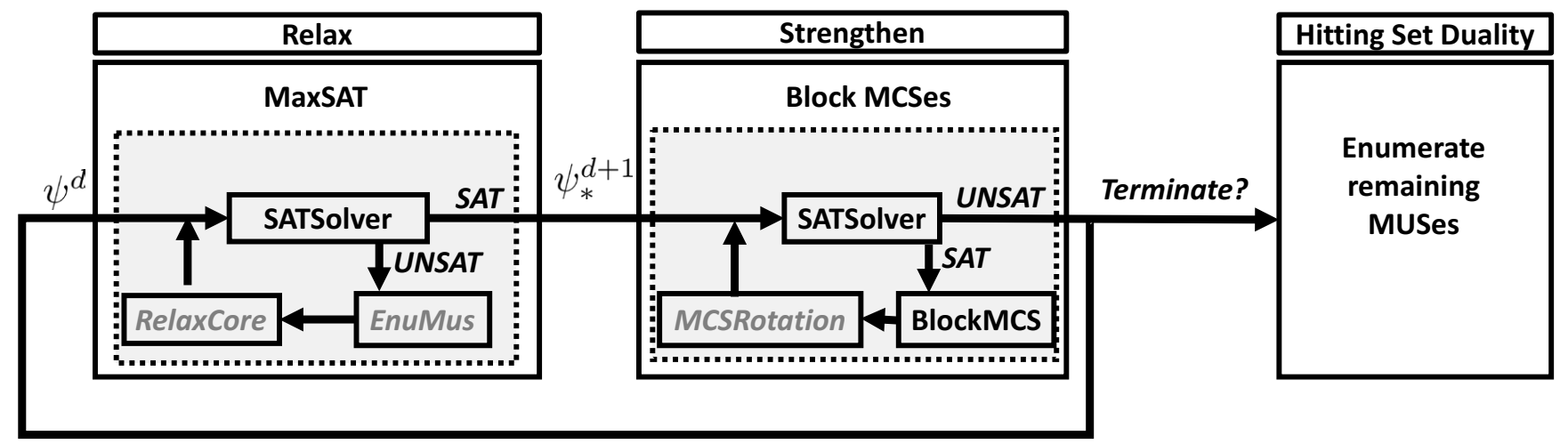

Figure 1: A schematic workflow of the relaxation and strengthening framework. We highlighted using italic gray new components of the framework that we propose in this work. RelaxCore contains additional constraints that connect projection and selector variables, EnuMus is a new MUS enumeration procedure, and MCSRotation is an MCS rotation based mechanism.

MCSes and MUSes found so far. Finally, cards stores all constraints produced by Relax. We will use a shortcut $\rho$ to denote $\wedge$ cards $\wedge$ map $\wedge$ projs. Using MAXSAT terminology, note that $\rho$ cannot be relaxed, e.g., they are 'hard' clauses, in contrast to $\psi^{d}$, that are called 'soft' clauses.
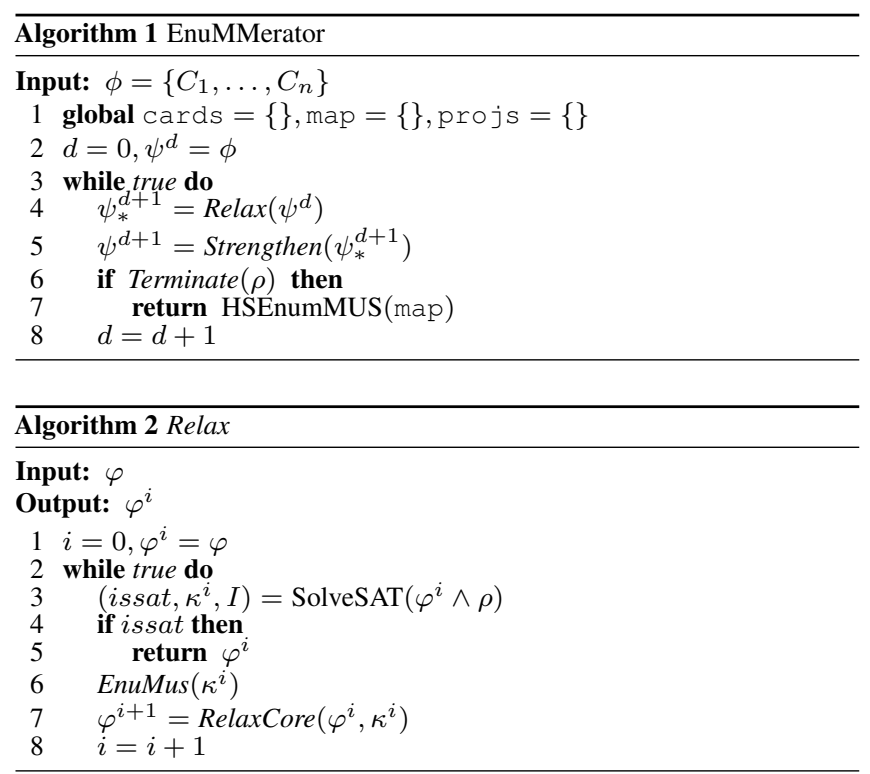

\section{Relax}

Algorithm 2 shows the Relax procedure, which extends PM1 [Fu and Malik, 2006]. Given an unsatisfiable formula $\varphi^{0} \wedge \rho$, Relax performs a sequence of its relaxations. The algorithm can only relax clauses in $\varphi^{i}$. At each step, it calls a SAT solver to check whether the current formula $\varphi^{i} \wedge \rho$ is satisfiable (line 3). If so, it terminates. Otherwise, it finds a core $\kappa^{i} \subseteq \varphi^{i}$, relaxes the formula $\varphi^{i}$ by relaxing clauses in $\kappa^{i}$ by calling RelaxCore in line 7 . On termination, the resulting formula $\varphi^{i}$ is such that $\varphi^{i} \wedge \rho$ is satisfiable. Moreover, each solution of $\varphi^{i} \wedge \rho$ is a MCS of minimal cardinality of $\varphi$ [Morgado et al., 2012].

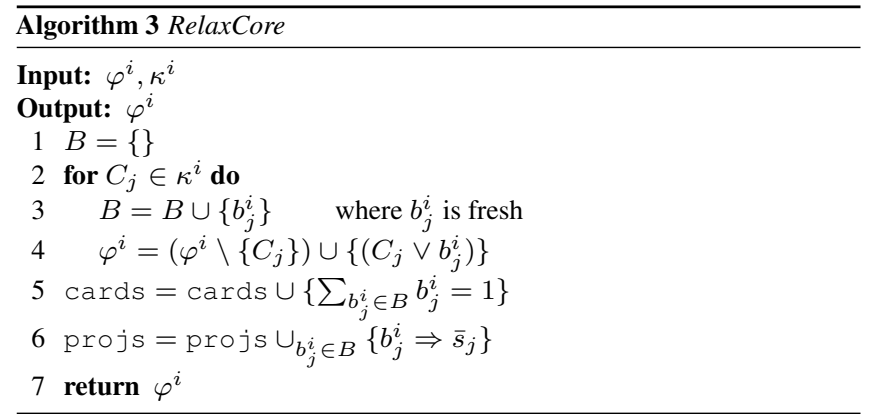

Next we consider the core relaxation procedure RelaxCore (Algorithm 3), which relaxes soft constraints $\varphi^{i}$ using the core $\kappa^{i}$. RelaxCore introduces a relaxation variable $b_{j}^{i}$ for each clause $C_{j} \in \kappa^{i}$ (lines 3-4). Then we add a cardinality constraint to ensure that exactly one of the relaxation variables can be set to true (line 5).

We extend the PM1 Relax in two ways. First, we enhance RelaxCore with a selector variable-based blocking technique. We implement a mapping from relaxation variables to selector variables using projs (line 6). Each time a new $b_{j}^{i}$ variable is added to a clause $C_{j}$, we connect $b_{j}^{i}$ with $s_{j}$ as $b_{j}^{i} \Rightarrow \bar{s}_{j}$. This guarantees that if a clause $C_{j}$ is disabled by $I$, i.e. $I\left(b_{j}^{i}\right)=$ $1, b_{j}^{i} \in C_{j}$, then the corresponding selector variable $s_{j}$ is set to false. Second, Relax uses a novel MUS enumerator, EnuMus (line 6), to extract a subset of MUSes from $\kappa^{i}$. Algorithm 5 will be discussed in Section 3.2.

Example 3. Consider the instance from Example 1. Suppose, in the first round, Relax finds a core $\kappa^{0}=\left(C_{1}, C_{2}, C_{3}\right)$ of $\varphi^{0}$. It relaxes this core using RelaxCore. Namely, three relaxation variables are introduced $\left\{b_{1}^{0}, b_{2}^{0}, b_{3}^{0}\right\}$ and the corresponding clauses are modified. We get $\varphi^{1}=\left\{C_{1} \vee b_{1}^{0}, C_{2} \vee\right.$ $\left.b_{2}^{0}, C_{3} \vee b_{3}^{0}, C_{4}, C_{5}\right\}$. Then we add the cardinality constraint $\left\{\sum_{j=1}^{3} b_{j}^{0}=1\right\}$ to cards. Finally, we add constraints $\left\{b_{j}^{0} \Rightarrow \bar{s}_{j}\right\}, j \in\{1,2,3\}$ to projs to connect relaxation variables to selector variables. The resulting formula $\varphi^{1} \wedge \rho$ (recall that $\rho=\operatorname{card} s \wedge \operatorname{map} \wedge$ projs) is satisfiable. 


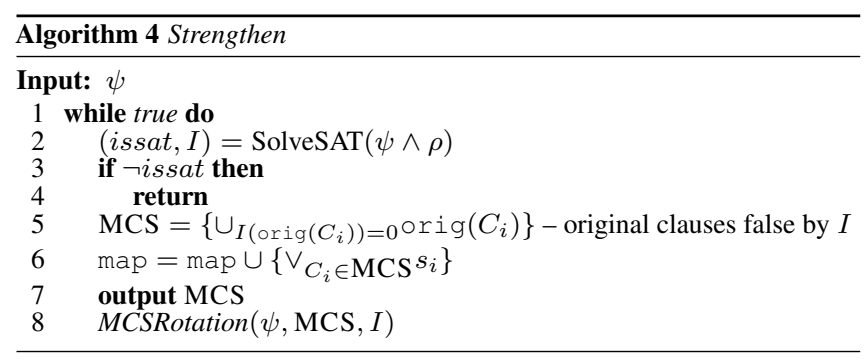

\section{Strengthen}

Strengthen enumerates all correction sets based on $\psi_{*}^{d+1} \wedge \rho$, until the strengthened $\rho$ renders the formula unsatisfiable. To do so, the procedure enumerates solutions of $\psi_{*}^{d+1} \wedge \rho$. For each solution $I$ it computes the corresponding MCS (line 5) and adds the correction set blocking clauses (recall (1), Section 2) to map (line 6). It uses a novel MCS rotation technique, MCSRotation, described in Section 3.3.

Example 4. We continue with the instance from Example 3. We recall that Relax returned a satisfiable formula $\psi_{*}^{1}=\varphi^{1} \wedge \rho$. Then Strengthen finds all solutions of $\psi_{*}^{1} \wedge \rho$. This formula has a solution where $b_{1}^{0}$ is assigned to 1 . Hence, $C_{1}$ is relaxed in this solution. This gives a minimal correction set $\left(C_{1}\right)$ and we block it by adding $\left(s_{1}\right)$ to map. This makes formula unsatisfiable finishing the first round.

\section{Terminate}

Terminate checks whether all MCSes have been found by checking satisfiability of $\rho$. If so, it enumerates remaining MUSes using the hitting set duality (HSEnumMUS) (line 7).

In [Morgado et al., 2012], it was shown that the relaxation and strengthening framework based on PM1 algorithm guarantees that (1) solutions of $\psi_{*}^{d+1} \wedge \rho$ are minimal correction sets and (2) MCSes are enumerated in the order of non-decreasing cardinality of these sets. As we use a different blocking technique for MCSes and, in addition, we block MUSes, we will need to prove these claims for EnuMMerator (see Section 3.4). Next we consider the remaining parts of EnuMMerator.

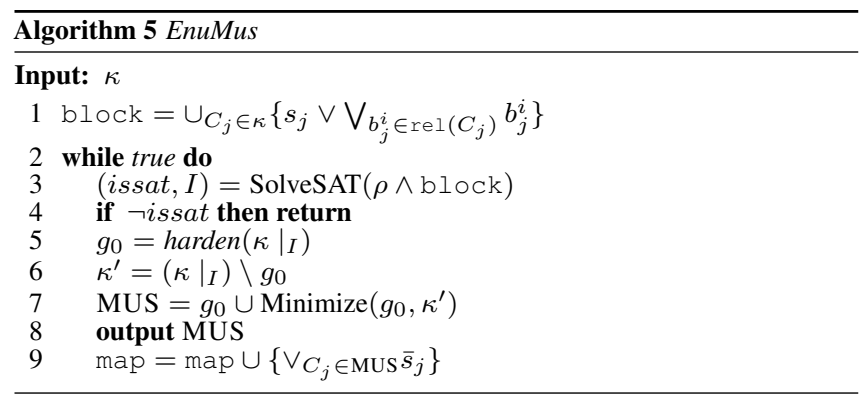

\subsection{A MUS Extractor}

In the core enumeration procedure, we need to extract cores of the original formula $\phi-$ the input formula to EnuMMerator. To do so, we will use cores $\kappa^{i}$ that are produced by Relax. We apply a useful notion of a projection to transform clauses in $\kappa^{i}$ to clauses of the original formula $\phi$ given an assignment $I$. Let $I$ be a solution of $\rho$ and $\kappa, \kappa=\kappa^{i}$, be a core produced at the $i$ th iteration of Relax. Define a projection as follows.

$$
\left.\kappa\right|_{I}=\left\{\operatorname{orig}\left(C_{j}\right) \mid C_{j} \in \kappa, \forall b_{j}^{i} \in \operatorname{rel}\left(C_{j}\right) I\left(b_{j}^{i}\right)=0\right\} .
$$

Intuitively, $\left.\kappa\right|_{I}$ is a subset of clauses of the original formula that are not satisfied by the solution $I$. [Bacchus and Narodytska, 2014] showed that given a core $\kappa$ produced by PM1-based Relax, the projection $\left.\kappa\right|_{I}$ is a core of the original formula $\phi$, where $I$ is a solution of cards. Let MUS-all be a set of all known MUSes.

First, we show that the same result holds for a solution of $\rho$. Second, we investigate relations between solutions of cards and solutions of $\rho=\operatorname{cards} \wedge \operatorname{map} \wedge$ projs. We demonstrate that core blocking constraints in map do not guarantee that $\left.\kappa\right|_{I}$ is not a superset of a known MUS in MUS-all. This is an undesirable property as we might keep finding known MUSes during enumeration. To fix the problem of projecting on known cores we introduce an additional set of constraints that are used only in EnuMus, block constraints.

Proposition 3.1. Let I be a solution of $\rho$. Then $\left.\kappa\right|_{I}$ is a core of the original formula $\phi$.

Proof. Suppose $\left.\kappa\right|_{I}$ is not a core. Let $I^{\prime}$ be solution of $\left.\kappa\right|_{I}$. We form a new solution $J$ as follows. If $v \in \operatorname{vars}\left(\left.\kappa\right|_{I}\right)$ then $J(v)=I^{\prime}(v)$, otherwise $J(v)=I(v)$. Next, we note that $\operatorname{vars}\left(\left.\kappa\right|_{I}\right) \cap \operatorname{var} s(\rho)=\{\}$. Hence, we do not change the assignment of projection and selector variables in $J$ compared to $I$. Note that clauses in $\kappa \backslash\left(\left.\kappa\right|_{I}\right)$ are satisfied using the corresponding projection variables $b_{j}^{i}$ that are set to 1 in $I$, hence, they are also set to 1 in $J .\left.\kappa\right|_{I}$ is satisfied as $\operatorname{var} s\left(\left.\kappa\right|_{I}\right)$ are assigned as in $I^{\prime}$. Finally, $\rho$ is satisfied as we did not change assignment of variables in $\rho$ in $J$ compared to $I$. Hence, $J$ is a solution of $\kappa \wedge \rho$, which leads to a contradiction.

The next example shows that map and projs are not sufficient to eliminate all known cores.

Example 5. We use the instance from Example 4. Consider the second round and assume that EnuMus was called in the first round and recorded the core it found in map. At this point, cards and projs are as in Example 4 and map = $\left\{\left(s_{1}\right),\left(\bar{s}_{1} \vee \bar{s}_{2} \vee \bar{s}_{3}\right)\right\}$. Suppose Relax finds a core $\kappa^{0}=$ $\left(C_{1} \vee b_{1}^{0}, C_{4}, C_{5}\right)$. EnuMus is called. Consider two solutions of $\rho: I_{1}=\left(b_{1}^{0}=0, b_{2}^{0}=1, b_{3}^{0}=0, s_{1}=1, s_{2}=\ldots=s_{5}=0\right)$, and $I_{2}=\left(b_{1}^{0}=0, b_{2}^{0}=0, b_{3}^{0}=1, s_{1}=1, s_{2}=\ldots=\right.$ $\left.s_{5}=0\right)$. These solutions are identical except for swapped values of $b_{2}^{0}$ and $b_{3}^{0}$. Suppose we find $I_{1}$ first and project it to get a core $\left.\kappa^{0}\right|_{I_{1}}=\left\{C_{1}, C_{4}, C_{5}\right\}$. We can block this core by adding the clause $\left(\bar{s}_{1} \vee \bar{s}_{4} \vee \bar{s}_{5}\right)$ to map. However, this clause does not eliminate $I_{2}$ that is projected to the same core: $\left.\kappa^{0}\right|_{I_{2}}=\left\{C_{1}, C_{4}, C_{5}\right\}$.

The main issue here is that $C_{4}$ and $C_{5}$ are always selected in a projection as they do not contain projection variables. However, there are no constraints to enforce that if a clause is in a projection then the corresponding selector variable is set to 1 . So, we cannot enforce that for any solution I if $C_{5} /\left.C_{4} \in \kappa^{0}\right|_{I}$ then $s_{5} / s_{4}$ must be 1 in this example.

From Example 5 it follows.

Proposition 3.2. There exists a solution I of $\rho$ such that $m \subseteq$ $\left.\kappa\right|_{I}, m \in M U S-a I l$.

Next we describe our MUS enumerator, EnuMus (Algorithm 5). EnuMus takes a core $\kappa$ as an input. By Proposition 3.1, we just need to enumerate solutions of $\rho$ to extract 
cores of the original formula $\phi$. EnuMus performs such enumeration. However, by Proposition 3.2 the enumeration procedure will produce a lot of solutions that correspond to MUSes that we have encountered before. We introduce additional constraints, block, to make sure that if a clause is in a projection, i.e. $\left.C_{j} \in \kappa\right|_{I}$, then the corresponding selector variable $s_{j}=1$ (line 1, EnuMus). block $=\cup_{C_{j} \in \kappa}\left\{s_{j} \vee \bigvee_{b_{j}^{i} \in \operatorname{rel}\left(C_{j}\right)} b_{j}^{i}\right\}$. Hence, if $\left.\kappa\right|_{I}$ is a superset of a known MUS $m$ then the core blocking clause $\left(\vee_{C_{j} \in m} \bar{s}_{j}\right)$ is falsified. The block constraints resolve the issue pointed out in Example 5.

Proposition 3.3. If I is a solution of $\rho$ then $\forall m \in M U S-a \perp I$ we have that $\left.m \nsubseteq \kappa\right|_{I}$.

Proof. Let $\pi=\left.\kappa\right|_{I}$. The proof follows from two observations. First, we know that $I\left(b_{j}^{i}\right)=0, \forall b_{j}^{i} \in \operatorname{rel}\left(C_{j}\right), C_{j} \in \pi$. Hence, block clauses enforce that $s_{j}=1$, which implies that $\left(\vee_{C_{j} \in \pi} \bar{s}_{j}\right)$ is falsified by $I$ and cannot be contained in map. As map blocks all MUSes that have been found so far, we know that $\pi$ is not contained in known MUSes.

Algorithm 5 shows pseudocode. EnuMus takes a core $\kappa$ as an input. Then it enumerates solutions of $\rho \wedge \mathrm{block}$. By Proposition 3.3, if there exists a solution $I$ of $\rho \wedge \mathrm{block}$ then $\left.\kappa\right|_{I}$ contains a new MUS of the original formula $\phi$. Before minimizing $\left.\kappa\right|_{I}$, we perform simple preprocessing based on the duality between cores and correction sets. The harden procedure (line 6) returns $g_{0}$ as the clauses that must be in the minimal core: $C \in\left(\left.\kappa\right|_{I}\right)$ s.t. $\operatorname{MCS} \cap\left(\left.\kappa\right|_{I}\right)=\{C\}$ for some MCS that we found so far. The same optimization was used in [Liffiton et al., 2016; Zielke and Kaufmann, 2015]. Finally, we minimize $\kappa^{\prime}$ with respect to $g_{0}$ as hard constraints and block it.

Example 6. We continue with the instance from Example 4. Consider the second round and assume that EnuMus was called in the first round and recorded the core it found in map. At this point, cards and projs are as in Example 4 and $\operatorname{map}=\left\{\left(s_{1}\right),\left(\bar{s}_{1} \vee \bar{s}_{2} \vee \bar{s}_{3}\right)\right\}$. Suppose Relax finds a core $\kappa^{0}=\left(C_{1} \vee b_{1}^{0}, C_{4}, C_{5}\right)$. EnuMus is called. We form block constraints: block $=\left\{\left(s_{4}\right),\left(s_{5}\right)\right\}$ as there are no projection variables in $C_{4} / C_{5}$. The first solution of $\left(\rho \wedge b_{10 c k}\right)$ is $I=$ $\left(b_{1}^{0}=0, b_{2}^{0}=1, b_{3}^{0}=0, s_{1}=1, s_{2}=s_{3}=0, s_{4}=s_{5}=1\right)$. The corresponding projection $\left.\kappa^{0}\right|_{I}=\left\{C_{1}, C_{4}, C_{5}\right\}$. We check that $\left.\kappa^{0}\right|_{I}$ is minimal and block this core by adding the clause $\left(\bar{s}_{1} \vee \bar{s}_{4} \vee \bar{s}_{5}\right)$ to map. Note that in contrast with Example 5, $s_{4}$ and $s_{5}$ must take values 1 due to block constraints. This allows us to eliminate solutions that are mapped to known MUSes, like $I_{2}$.

\subsection{Enhanced MCS Extractor}

In this section we consider a new approach to speed up the MCS enumeration procedure. Strengthen takes $\psi$ as the input and finds all solutions. However, the size of the formula grows at each iteration and finding new solutions becomes expensive. Here we propose a novel approach to speed up MCS enumeration which is very efficient in practice.

We will show in Section 3.4 that EnuMMerator finds MCSes of non-decreasing cardinalities of the original formula $\phi$. Let MCS-a $l_{<k}$ be a set of all MCSes of $\phi$ of size less than $k$.
Proposition 3.4. Let $m$ be a correction set of size $k$. The correction set $m$ is minimal iff $\forall \mathrm{MCS} \in M C S-a I_{<k}$ MCS $\not \subset m$.

Proof. As $m$ is not a superset of any MCS of size smaller than $k$, then it is minimal by definition.

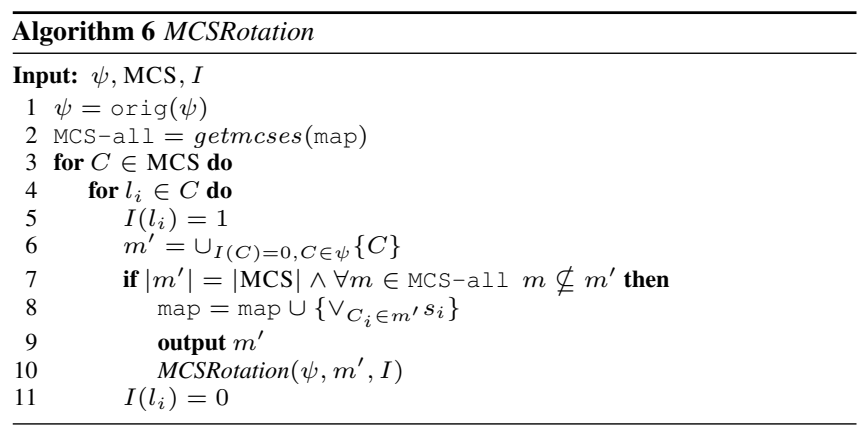

Algorithm 6 shows pseudocode. We first obtain the original formula by removing all relaxation variables from it (line 1) and get all MCSes from the map using getmcses(map) (line 2). In practice, we keep all MCSes in a data structure so we do not need to extract MCSes from map each time. Our rotation is inspired by to the model rotation techniques from [Belov and Marques-Silva, 2011] which is an efficient technique for core minimization. We start from an assignment $I$ and the corresponding MCS. We pick a clause $C$ from MCS and a literal $l_{i} \in C$. As $C$ is in the MCS, then $I(C)=0$. Then we satisfy a literal $l_{i}$ by flipping $I\left(l_{i}\right)=0$ to $I\left(l_{i}\right)=1$ (line 5). This makes $C$ satisfiable but other clauses might become violated. We compute a correction set $m^{\prime}$ that corresponds to a new assignment (line 6). If the size of $\mathrm{m}^{\prime}$ is equal to the size of MCS and $\forall m \in \mathrm{MCS}-\mathrm{al} 1, m$ is not subset of $m^{\prime}$ then, by Proposition 3.4, we know that $m^{\prime}$ is a MCS (line 7). Then we recursively call MCSRotation (line 10).

Example 7. We continue with the instance from Example 4. We focus on Strengthen at the second round. Recall that Relax returned a satisfiable formula $\psi_{*}^{2}=\varphi^{1} \wedge \rho$. This formula has four solutions that correspond to MCSes: $\left(C_{2}, C_{4}\right)$, $\left(C_{2}, C_{5}\right),\left(C_{3}, C_{4}\right)$ and $\left(C_{3}, C_{5}\right)$. So the Strengthen algorithm has to make 4 SAT calls to find them. With MCSRotation, Strengthen can reduce the number of calls to the SAT solver. Suppose Strengthen finds the first solution I and passes it to MCSRotation. Suppose $I=\left(x_{1}=1, x_{2}=0, x_{3}=0\right)$ and the corresponding MCS is $\left\{C_{2}, C_{4}\right\}$ (see Example 1 for the definition of clauses). At this point $M C S-a 1 I_{\leq 2}=\left\{\left(C_{1}\right)\right\}$. Suppose we flip $x_{2}=0$ to $x_{2}=1$. The new assignment violates $m^{\prime}=\left\{C_{3}, C_{4}\right\}$. As $\left|m^{\prime}\right|=2$ we check that none of MCSes in MCS-aI $I_{\leq 2}$ is a subset of $m^{\prime}$. Hence, we know that $m^{\prime}$ is a new MCS without calling a SAT solver.

\subsection{Correctness}

In this section, we sketch a proof of correctness.

Theorem 3.5. EnuMMerator finds all MCSes and MUSes when it terminates.

Proof. (Sketch) We first prove correctness of a simpler version of EnuMMerator where EnuMus is omitted. We recall 
that map contains two types of blocking clauses: map = mapmcs $\cup$ mapmus. If we exclude EnuMus, we have that map $=$ mapmcs.

A simplified EnuMMerator enumerates all MCSes and uses hitting set duality to enumerate all MUSes. Hence, we can reuse the proof of correctness of the the relaxation and strengthening framework for finding MCSes as in [Morgado et al., 2012]. We only need to prove correctness of our MCS blocking procedure and the termination condition.

The main part to prove is that we never block a MCS that we have not produced. Consider the $i$ th iteration. We prove this by contradiction. Suppose, there exists a MCS $m$ that has never been encountered during the execution of EnuMMerator. Suppose, it was eliminated after adding a projection constraint to projs at the $i$ th iteration. So, we have that $\psi^{d+1} \wedge \rho$ has a solution $I$ that correspond to $m$ and $\psi^{d+1} \wedge \rho \wedge\left(b_{j}^{i} \Rightarrow \bar{s}_{j}\right)$ eliminates this solution. We form a new solution $I^{\prime}=I$ and set $I^{\prime}\left(s_{j}\right)=1$. Note that $I^{\prime}$ must satisfy $\psi^{d+1} \wedge \rho$ as setting $s_{j}$ to 1 will not violate any constraint. It also satisfies $b_{j}^{i} \Rightarrow \bar{s}_{j}$ as $I\left(s_{j}\right)=1$. On the other hand, $I^{\prime}$ corresponds to the same MCS $m$, as the assignment of projection variables does not change. Therefore, $b_{j}^{i} \Rightarrow \bar{s}_{j}$ cannot eliminate $m$. Hence, it should be eliminated by the correction set blocking clauses (1) at the $i$ th iteration on seeing MCS $m^{\prime}$. Note that the clause $\vee_{C_{i} \in m^{\prime}} s_{i}$ we add to mapmcs only eliminates $m^{\prime}$ and its supersets. Hence, either $m=m^{\prime}$ or it is not minimal. This leads to a contradiction.

Next we come back to EnuMMerator with EnuMus. We prove that EnuMus does not change the solution space of $\psi^{d} \wedge$ cards $\wedge$ projs $\wedge$ mapmcs. Namely, we prove that any solution $\psi^{d} \wedge$ cards $\wedge$ projs $\wedge$ mapmcs can be extended to a solution of mapmus. Let $I$ be a solution of $\psi^{d} \wedge$ cards $\wedge$ projs $\wedge$ mapmcs. Consider an MCS of the original formula $\phi$ that corresponds to this solution $I$. First, we note that there exists $b_{j}^{i} \in \operatorname{rel}\left(C_{j}\right), C_{j} \in \mathrm{MCS}$ such that $I\left(b_{j}^{i}\right)=1$. Then, $I\left(s_{j}\right)$ must be 0 for all $j$ such that $C_{j} \in \mathrm{MCS}$ due to projs clauses. The main observation is that any MCS hits all MUSes. Therefore, for each clause $\left(\bar{s}_{j_{1}} \vee \ldots \bar{s}_{j_{n}}\right) \in$ mapmus, at least one of the literals is set to 0 . Hence, all clauses in mapmus are satisfied.

\section{Related Work}

We briefly overview two types of closely related work. The first line of related research proposed a relaxation and strengthening framework for MCS enumeration [Marques-Silva and Planes, 2008; Morgado et al., 2012; Alviano, 2017b]. The Relax procedure was performed using MSU3, PM1 and OLL MAXSAT algorithms respectively. We use the same framework but propose a different blocking technique that allows us to enumerate cores as well as correction sets. Moreover, we propose a procedure to perform core extraction and MCS rotation to speed up MCS enumeration.

The second line of work develops methods that work with the original formula [Liffiton and Sakallah, 2008a; Bacchus et al., 2014; Zielke and Kaufmann, 2015; Bacchus and Katsirelos, 2016; Previti et al., 2017]. Our approach is based on ideas from the MARCO algorithm that explores a lattice of all possible subsets of clauses. However, instead of explicit exploration of points of the lattice, we extract sets of points at each iteration. These sets come from solutions of satisfiability problems that we either build incrementally (for MCSes) or form dynamically (for MUSes) during search.

A number of algorithms were proposed that focus on MCS or MUS enumeration. [Previti et al., 2017] proposed keeping track of unsatisfiable cores using a new caching mechanism, and proposed an efficient way to check if a given sub-formula contains a known core. It is a matter of future research to explore if we can use a caching mechanism within the relaxation and strengthening framework. It will likely be different from [Previti et al., 2017], as we do not grow a maximal satisfying assignment to obtain MCSes. [Bacchus et al., 2014] proposed a relaxation search that uses a SAT solver to efficiently find sets of optional clauses to remove. [Zielke and Kaufmann, 2015] proposed to consider the block property of a set of clauses. These clauses either occur together in a MUS or do not occur at all. The discovered sets with this property can be used to speed up MARCO. [Bacchus and Katsirelos, 2016] show that MARCO can be significantly improved by incrementally enumerating cores and proposed other improvements. They demonstrated that any MARCO-like algorithm can leverage incremental core enumeration if it is deeply integrated with MCSMUS. The key idea behind the incremental part is that MCSMUS can take advantage of enumerated MCSes to produce MUSes incrementally. Such deep integration is one possible avenue for improving our algorithm.

\section{Experimental Evaluation}

We work with a standard set of 295 benchmarks from the MUS track of the 2011 SAT competition [Jarvisalo et al., 2011]. We ran our experiments on Intel(R) Xeon(R) 3.50GHz. We compare our algorithm, called FLINT, with MARCO, the stateof-the-art method that simultaneously extracts MUSes and MCSes. We build FLINT on top of PM1 from [Janota, 2013]. For fair comparison, we call the MUSer algorithm [Belov and Marques-Silva, 2012] as a standalone procedure for core minimization the same way as MARCO. As our algorithm can also be geared toward correction sets enumeration, we consider a version that only enumerates correction sets (FLINT-MCS) and we compare it with MARCO-MCS (called MARCO-MSS in [Liffiton et al., 2016]), which is a version of MARCO biased toward MCS enumeration. Finally, we compare with several algorithms that focus on enumeration of only MUSes/MCSes. We use timeout of $3600 \mathrm{sec}$ in all runs.

We discuss implementation details. First, we consider how we preform the forward subsumption check in line 7 of Algorithm 6. Recall that it suffices to perform only forward subsumption as none of the existing MCSes is a superset of a candidate correction set $m$. In the benchmark set, the size of correction sets that we find is on average 5, and generally less than 10. So instead of using a general discrimination tree index or index based on feature vectors [Schulz, 2013], it suffices to index each MCS of length $n$ into a table (trie or hash-table) that contains all size $n$ sets. Then $\left(\begin{array}{c}|m| \\ n\end{array}\right)$ lookups are performed for every $n=1, \ldots,|m|$. In our experiments we used a trie to store MCSes [Tessil, 2017]. Second, we discuss the MUS extractor. We found that $\kappa^{\prime}$ (Algorithm 5, 


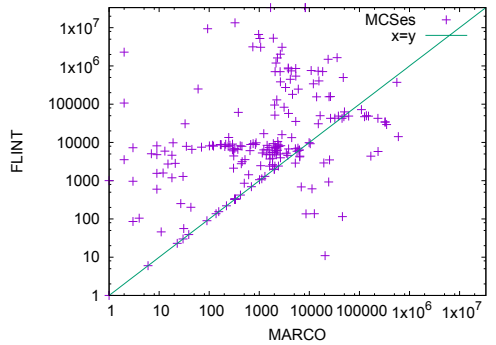

(a)

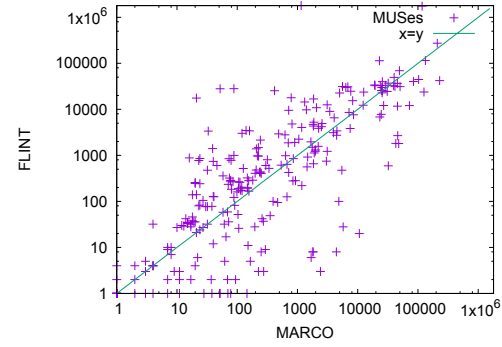

(b)

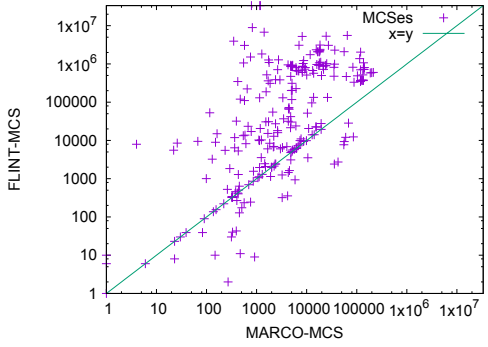

(c)

Figure 2: Comparison of FLINT and MARCO algorithms: (a) the number of MCSes produced by FLINT and MARCO; (b) the number of MUSes produced by FLINT and MARCO; (c) the number of MCSes produced by FLINT-MCS and MARCO-MCS.

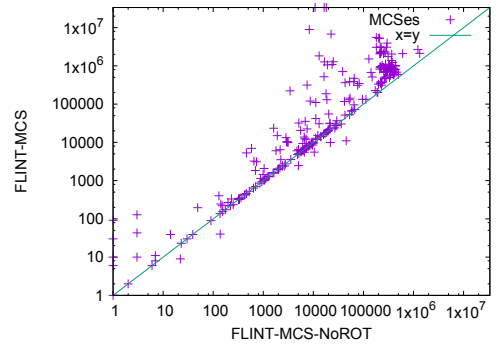

(a)

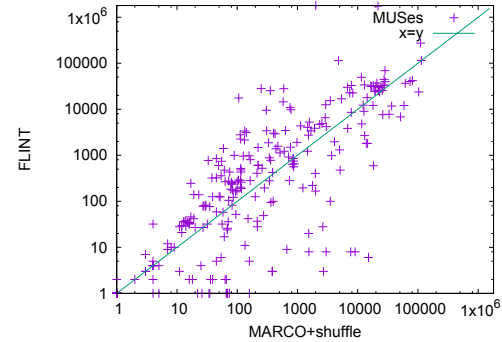

(b)

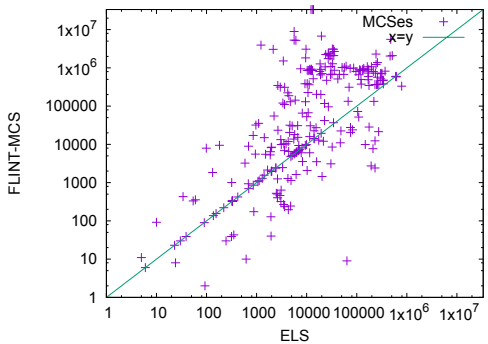

(c)

Figure 3: (a) Comparison of FLINT-MCS and FLINT-MCS-noROT modification algorithms that does not use MCSRotation in terms of the number of MCSes; (b) Comparison of FLINT and MARCO+shuffle heuristics in terms of the number of MUSes; (c) Comparison of FLINT-MCS and ELS algorithms in terms of the number of MCSes.

line 6) often contains multiple minimal cores. This might be related to the fact that the input core $\kappa$ is not minimal as it is very expensive to minimize it. However, MUSer only finds one core per call. We employ a simple heuristic where we randomly shuffle clauses in $\kappa^{\prime}$ and give it to MUSer again as it often produces different cores. We filter out repeated cores and terminate shuffling if we start encountering many known MUSes (five in a row in our implementation). Since this algorithm relies on randomization, we run it five times and show the average result.

\subsection{Comparison with MARCO}

We compare our algorithm with MARCO. First we compare the algorithms by number of MUSes and MCSes generated within 3600s. Figures (2a)-(2b) show the corresponding log scale scatter plots. In all plots, the more points are above the $x=y$ line the better FLINT/FLINT-MCS performs compared to the competing algorithm. As can be seen from these plots, FLINT outperforms MARCO in the enumeration of both objects (more points above the $x=y$ line indicates that FLINT outperforms MARCO). FLINT finds more MCSes in 166 instances and more MUSes in 143 instances compared to MARCO. On the other hand, MARCO finds more MCS in 54 instances and more MUSes in 97 instances compared to FLINT. The algorithms ties on the remaining instances. We also compared 'biased' to the MCSes versions of FLINT and MARCO (see Figures (2c)). Again, we observe that FLINT-MCS performs much better com- pared to MARCO-MCS: FLINT-MCS finds more MCS in 202 instances and fewer MCSes in 55 instances compared to MARCO-MCS. We also investigate the contribution of the MCSRotation procedure to the performance of FLINT-MCS (Figure 3a). To do so, we run FLINT-MCS with and without MCS rotation. MCSRotation helps in most instances (187 instances) and hurts in only a small fraction of instances (22 instances). Finally, we compare with MARCO enhanced with the same shuffling heuristics for MUS extraction as used in our algorithm. To do so, each time we get a seed which results to unsatisfiable subformula, the shuffle clauses in this seed and pass it to MUSer. If we encounter five known MUSes in a row we terminate stop shuffling. Figure $3 \mathrm{~b}$ shows the results. We found that the shuffling heuristics slightly degrades performance of MARCO. For example, FLINT finds more MUSes in 153 instances compared to MARCO+shuffling variants.

\subsection{Comparison with Other Algorithms}

We compare FLINT-MCS with state-of-the-art algorithms that focus on enumeration of only one type of objects: MCSes or MUSes. First, we consider several state-of-the-art algorithms for MCS enumeration which are ELS and CLD for propositional formulas and CIRCUMSCRIPTINO for propositional theories. To compare with ELS and CDL, we use the same standard set of benchmarks. FLINT-MCS finds more MCSes in 167 instances, fewer in 76 instances compared to ELS and tie on the remaining instances (Figure 3c). FLINT-MCS finds more MCSes in 173 instances, fewer in 65 instances com- 


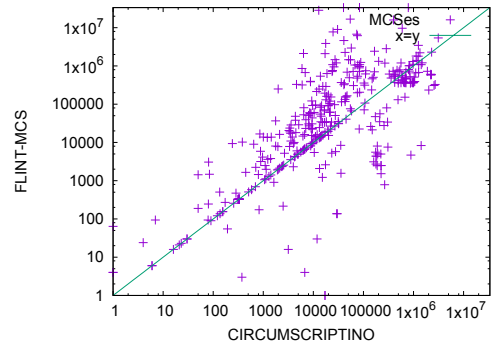

(a)

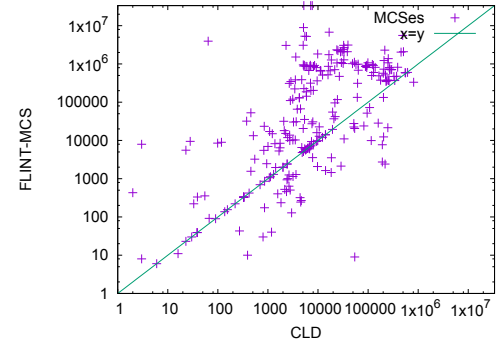

(b)

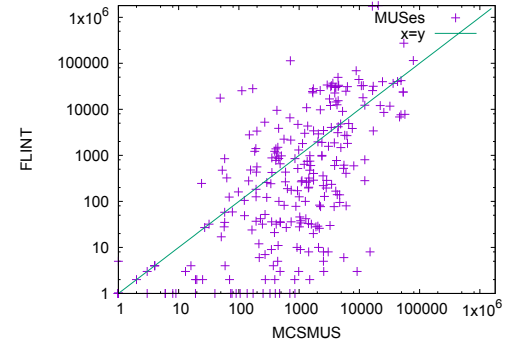

(c)

Figure 4: (a) Comparison of FLINT-MCS and CIRCUMSCRIPTINO in terms of the number of MCSes; (b) Comparison of FLINT-MCS and CLD in terms of the number of MCSes; (c) Comparison of FLINT-MCS and MCSMUS in terms of the number of MUSes.

pared to CLD and tie on the remaining instances (Figure 4b). In case of CIRCUMSCRIPTINO, we used a different dataset as it does not accept the DIMACS CNF input format; hence we compared on the benchmark set provided by the authors that contains instances encoded as a propositional theory and as DIMACS CNF [Alviano, 2017a]. The total number of instances in 395 in this dataset. FLINT-MCS finds more MCSes in 210 instance and fewer MCSes in 99 instances compared to CIRCUMSCRIPTINO (Figure 4a).

Overall, these results show that the MCS-biased version of FLINT performs very well compared to algorithms that only focus on MCSes. We also compare FLINT with the MCSMUS algorithm, that focuses on MUS enumeration [Bacchus and Katsirelos, 2015; Bacchus and Katsirelos, 2016]. As the non-incremental version of this algorithm is less efficient than MARCO [Bacchus and Katsirelos, 2015], we do not consider it here. Instead, we consider a version of MCSMUS that computes MUSes incrementally. FLINT finds more MUSes in 85 and fewer MUSes in 171 instances compared to MCSMUS (Figure 4c). This shows that FLINT is competitive with an incremental MCSMUS. It also shows that our algorithm can potentially be improved by deep integration of incremental core enumeration [Bacchus and Katsirelos, 2016].

\subsection{Discussion of Results}

We discuss how our algorithm can be extended based on several observations from our experiments.

As the solver is based on the relaxation and strengthening framework, the choice of the relaxation technique that is borrowed from MAXSAT has strong influence on the performance. Currently, we use the PM1 algorithm, which is easier to implement. However, we observed that in the benchmark set, there are 32 instances where we were not able to complete the first relaxation step using PM1. A more efficient relaxation procedure might help on these instances. The PM1 algorithm can be replaced with other cardinality-based relaxation techniques, e.g. MAXRES, OLL, ONE, etc, [Andres et al., 2012; Narodytska and Bacchus, 2014; Alviano et al., 2015]. Common to these algorithms is the requirement to keep track of all introduced relaxation constraints and make sure that these constraints satisfy Proposition 3.1.

Core minimization is an important and, often, the most expensive part. Several improvements over FLINT are possible.
The feedback loop between cores and correction sets can be improved. While the harden procedure [Previti et al., 2017; Zielke and Kaufmann, 2015] is a simplistic preprocessing step, yet we observed it can identify up to $30 \%$ of clauses in $\kappa$ as necessarily members of the cores. To this end, it is interesting to build a solver with deep integration of MCSMUS as described in [Bacchus and Katsirelos, 2016]. Finally, it should be possible to take advantage of $\kappa^{\prime}$ containing multiple cores produced by Relax. We observed that a lot of cores produced from $\kappa^{\prime}$ share a large number of clauses. This can be exploited in core minimization.

\section{Conclusion}

In this work, we proposed an algorithm to enumerate minimal correction sets and cores simultaneously. We combined the relaxation and strengthening framework with lattice exploration methods. We propose several new techniques that allow efficient core and correction set enumeration. Our PM1 based prototype outperforms MARCO in enumeration of MUSes and MCSes on a standard set of benchmarks.

\section{References}

[Alviano et al., 2015] Mario Alviano, Carmine Dodaro, and Francesco Ricca. A maxsat algorithm using cardinality constraints of bounded size. In Proceedings of the TwentyFourth International Joint Conference on Artificial Intelligence, IJCAI 2015, pages 2677-2683. AAAI Press, 2015.

[Alviano, 2017a] Mario Alviano. Circumscriptino. https://alviano.com/software/circumscriptino/, 2017.

[Alviano, 2017b] Mario Alviano. Model enumeration in propositional circumscription via unsatisfiable core analysis. TPLP, 17(5-6):708-725, 2017.

[Andres et al., 2012] Benjamin Andres, Benjamin Kaufmann, Oliver Matheis, and Torsten Schaub. Unsatisfiability-based optimization in clasp. In Technical Communications of the 28th International Conference on Logic Programming, ICLP 2012, volume 17, pages 211-221, 2012.

[Bacchus and Katsirelos, 2015] Fahiem Bacchus and George Katsirelos. Using minimal correction sets to more efficiently compute minimal unsatisfiable sets. In Computer Aided Verification - 27th International Conference, CAV 2015, July 18-24, 2015, volume 9207, pages 70-86, 2015. 
[Bacchus and Katsirelos, 2016] Fahiem Bacchus and George Katsirelos. Finding a collection of muses incrementally. In Integration of AI and OR Techniques in Constraint Programming - 13th International Conference, CPAIOR 2016, volume 9676, pages 35-44, 2016.

[Bacchus and Narodytska, 2014] Fahiem Bacchus and Nina Narodytska. Cores in core based maxsat algorithms: An analysis. In Theory and Applications of Satisfiability Testing - SAT 2014 - 17th International Conference, Held as Part of the Vienna Summer of Logic, VSL 2014, volume 8561, pages 7-15, 2014.

[Bacchus et al., 2014] Fahiem Bacchus, Jessica Davies, Maria Tsimpoukelli, and George Katsirelos. Relaxation search: A simple way of managing optional clauses. In Proceedings of the Twenty-Eighth AAAI Conference on Artificial Intelligence, 2014, pages 835-841, 2014.

[Belov and Marques-Silva, 2011] Anton Belov and Joao Marques-Silva. Accelerating MUS extraction with recursive model rotation. In International Conference on Formal Methods in Computer-Aided Design, FMCAD '11, 2011, pages 37-40. FMCAD Inc., 2011.

[Belov and Marques-Silva, 2012] Anton Belov and Joao Marques-Silva. Muser2: An efficient MUS extractor. JSAT, 8(3/4):123-128, 2012.

[Belov et al., 2012] Anton Belov, Ines Lynce, and Joao Marques-Silva. Towards efficient MUS extraction. AI Commun., 25(2):97-116, 2012.

[Fu and Malik, 2006] Zhaohui Fu and Sharad Malik. On solving the partial MAX-SAT problem. In Theory and Applications of Satisfiability Testing - SAT 2006, 9th International Conference, 2006, volume 4121, pages 252-265, 2006.

[Janota, 2013] Mikoas Janota. The MiFuMaX solver. http://sat.inesc-id.pt/ mikolas/sw/mifumax/, 2013.

[Jarvisalo et al., 2011] Matti Jarvisalo, Daniel Le Berre, Joao Marques-Silva, and Olivier Roussel. The MUS track of the 2011 SAT competition. http://www.satcompetition.org/2011/, 2011.

[Junker, 2004] Ulrich Junker. QUICKXPLAIN: preferred explanations and relaxations for over-constrained problems. In Proceedings of the Nineteenth National Conference on Artificial Intelligence, pages 167-172, 2004.

[Liffiton and Sakallah, 2008a] Mark H. Liffiton and Karem A. Sakallah. Algorithms for computing minimal unsatisfiable subsets of constraints. J. Autom. Reasoning, 40(1):1-33, 2008.

[Liffiton and Sakallah, 2008b] Mark H. Liffiton and Karem A. Sakallah. Reveal: A formal verification tool for verilog designs. In Logic for Programming, Artificial Intelligence, and Reasoning, 15th International Conference, LPAR 2008, Doha, Qatar, November 22-27, 2008, volume 5330, pages 343-352, 2008.

[Liffiton and Sakallah, 2009] Mark H. Liffiton and Karem A. Sakallah. Generalizing core-guided max-sat. In Theory and Applications of Satisfiability Testing - SAT 2009, 12th
International Conference, SAT 2009, volume 5584, pages 481-494. Springer, 2009.

[Liffiton et al., 2016] Mark H. Liffiton, Alessandro Previti, Ammar Malik, and Joao Marques-Silva. Fast, flexible MUS enumeration. Constraints, 21(2):223-250, 2016.

[Marques-Silva and Planes, 2008] Joao Marques-Silva and Jordi Planes. Algorithms for maximum satisfiability using unsatisfiable cores. In Design, Automation and Test in Europe, DATE 2008, Munich, Germany, March 10-14, 2008, pages 408-413. ACM, 2008.

[Marques-Silva et al., 2013] Joao Marques-Silva, Federico Heras, Mikolas Janota, Alessandro Previti, and Anton Belov. On computing minimal correction subsets. In IJCAI 2013, Proceedings of the 23rd International Joint Conference on Artificial Intelligence 2013, pages 615-622, 2013.

[Morgado et al., 2012] Antonio Morgado, Mark H. Liffiton, and Joao Marques-Silva. Maxsat-based MCS enumeration. In HVC 2012, Haifa, Israel, November 6-8, 2012. Revised Selected Papers, volume 7857, pages 86-101. Springer, 2012.

[Narodytska and Bacchus, 2014] Nina Narodytska and Fahiem Bacchus. Maximum satisfiability using coreguided maxsat resolution. In Proceedings of the Twenty-Eighth AAAI Conference on Artificial Intelligence, 2014., pages 2717-2723. AAAI Press, 2014.

[Previti et al., 2017] Alessandro Previti, Carlos Mencia, Matti Jarvisalo, and Joao Marques-Silva. Improving MCS enumeration via caching. In Theory and Applications of Satisfiability Testing - SAT 2017 - 20th International Conference, 2017, volume 10491, pages 184-194, 2017.

[Previti et al., 2018] Alessandro Previti, Carlos Mencia, Matti Jarvisalo, and Joao Marques-Silva. Premise set caching for enumerating minimal correction subsets. In Proceedings of the Thirty-Second AAAI Conference on Artificial Intelligence, 2018, 2018.

[Reiter, 1987] Raymond Reiter. A theory of diagnosis from first principles. Artif. Intell., 32(1):57-95, 1987.

[Schulz, 2013] Stephan Schulz. Simple and efficient clause subsumption with feature vector indexing. In Automated Reasoning and Mathematics - Essays in Memory of William W. McCune, volume 7788, pages 45-67, 2013.

[Tessil, 2017] Thibaut Tessil. A C++ implementation of a fast and memory efficient HAT-trie. https://github.com/Tessil/hat-trie/, 2017.

[Zhao and Liffiton, 2016] Wenting Zhao and Mark H. Liffiton. Parallelizing partial MUS enumeration. In 28th IEEE International Conference on Tools with Artificial Intelligence, ICTAI 2016, pages 464-471. IEEE Computer Society, 2016.

[Zielke and Kaufmann, 2015] Christian Zielke and Michael Kaufmann. A new approach to partial MUS enumeration. In Theory and Applications of Satisfiability Testing - SAT 2015 - 18th International Conference, 2015, volume 9340, pages 387-404, 2015. 